\title{
Primera aproximación de carga animal óptima en cuyes al pastoreo durante la época lluviosa en la sierra peruana
}

\author{
First approximation of optimum stocking rate in guinea pigs grazing during the \\ raining season in the Peruvian highlands
}

\author{
Liz Anaya L. ${ }^{1}$, Amparo Huamán C. ${ }^{1}$, Felipe San Martín H.², Miguel Ara G. ${ }^{2}$, \\ Fernando Carcelén C. ${ }^{2}$, Ronald Jiménez A. ${ }^{1,3}$
}

\section{Resumen}

\begin{abstract}
Se realizó una primera aproximación de la carga animal óptima en cuyes al pastoreo durante la época lluviosa en la sierra del Perú, utilizando una pastura perenne asociada conformada por Lolium multiflorum, Lolium perenne, Dactylis glomerata, Trifolium pratense, Trifolium repens y Medicago sativa en función a la ganancia de peso vivo individual y por unidad de área, consumo de alimento, conversión alimenticia, rendimiento de pastura, costos de producción y ratio beneficio costo. Se emplearon 132 cuyes machos $\mathrm{G}$ de 15 días de edad en un diseño de bloques completos al azar con tres ciclos de engorde de 60 días como bloque y cuatro tratamientos correspondientes a cargas de 400 (T1), 500 (T2), 600 (T3) y 700 (T4) cuyes/ha/año. Se trabajó en áreas de 200 $\mathrm{m}^{2}$ de pastura donde pastorearon grupos de 8, 10, 12 y 14 cuyes en T1, T2, T3 y T4,
\end{abstract}

\footnotetext{
${ }^{1}$ Grupo de Investigación Sistemas Productivos Sostenibles en Altura, Estación IVITA El Mantaro, Facultad de Medicina Veterinaria, Universidad Nacional Mayor de San Marcos, Jauja, Perú

${ }^{2}$ Grupo de Investigación Nutrición y Alimentación Animal, Laboratorio de Bioquímica, Nutrición y Alimentación Animal, Facultad de Medicina Veterinaria, Universidad Nacional Mayor de San Marcos, Lima, Perú

${ }^{3}$ E-mail:rjimeneza@unmsm.edu.pe
}

El estudio es parte del Proyecto A17081981, aprobado con Resolución Rectoral 04274-R-17 y financiado por el Programa de Proyectos de Investigación para Grupos de Investigación, Vicerrectorado de Investigación y Posgrado de la Universidad Nacional Mayor de San Marcos

Recibido: 21 de enero de 2021

Aceptado para publicación: 9 de octubre de 2021

Publicado: 22 de diciembre de 2021

CLos autores. Este artículo es publicado por la Rev Inv Vet Perú de la Facultad de Medicina Veterinaria, Universidad Nacional Mayor de San Marcos. Este es un artículo de acceso abierto, distribuido bajo los términos de la licencia Creative Commons Atribución 4.0 Internacional (CC BY 4.0) [https:// creativecommons.org/licenses/by/4.0/deed.es] que permite el uso, distribución y reproducción en cualquier medio, siempre que la obra original sea debidamente citada de su fuente original 
respectivamente. Los resultados demostraron que no hubo efecto de la carga animal sobre la ganancia de peso individual, consumo de alimento, conversión alimenticia y rendimiento de pastura $(\mathrm{p}>0.05)$. Los efectos sobre la ganancia por unidad de área y ratio beneficio costo $(p<0.05)$ fueron consecuencia directa y esperada del diferente número de animales entre cargas animales. El rango de carga animal fue insuficiente para determinar una carga óptima, de manera que tentativamente se sugiere utilizar la carga de 700 cuyes/ ha/año, por ser la carga que favorece lograr más cuyes con parámetros productivos similares a cargas menores y mejores parámetros económicos.

Palabras clave: cuy, pastoreo, carga animal óptima, pastura asociada, sierra peruana

\section{Abstract}

The present study carried out a first approximation of the optimum stocking rate in guinea pigs grazing during the rainy season in the Peruvian highlands, using an associated perennial pasture composed of Lolium multiflorum, Lolium perenne, Dactylis glomerata, Trifolium pratense, Trifolium repens y Medicago sativa based on individual body weight gain and per area, feed intake, feed conversion, pasture yield, production costs and cost benefit ratio. In total, 132 15-day-old male $G$ guinea pigs were used in a randomized complete block design with three 60-day fattening cycles as a block and four treatments corresponding to stocking rates of 400 (T1), 500 (T2), 600 (T3) and 700 (T4) guinea pigs/ ha/year. It was used areas of $200 \mathrm{~m}^{2}$ of pasture where groups of 8, 10,12 and 14 guinea pigs grazed in T1, T2, T3 and T4, respectively. The results showed that there was no effect of the stocking rate on individual body weight gain, feed intake, feed conversion and pasture performance yield $(>0.05)$. The effects on the gain per unit area and cost benefit ratio $(p<0.05)$ were a direct and expected consequence of the different number of animals between stockings. The range of stocking rates was insufficient to determine an optimal stocking rate, so it is tentatively suggested to use the stocking rate of 700 guinea $\mathrm{pigs} / \mathrm{ha} / \mathrm{year}$, as it is the stocking rate that allows more animals per area with productive parameters similar to lower stocking rates and better economic parameters.

Key words: guinea pigs, grazing, optimum stocking rate, associate pasture, Peruvian highlands

\section{INTRODUCCIÓN}

En el Perú, la crianza de cuyes se ha desarrollado enormemente, especialmente en la sierra. Según la última Encuesta Nacional Agropecuaria (INEI, 2017) se tiene 17 millones de cuyes (Cavia porcellus); esto es, $37 \%$ superior a la cifra de 2012 (INEI, 2012), especialmente debido a las ventajas de fertilidad, prolificidad y rápido crecimiento (Chauca, 1997).
Los cuyes son animales herbívoros y la mayoría de los sistemas de producción dedicados a esta especie utilizan altas proporciones de pastos en su alimentación porque cubre gran parte de sus requerimientos nutricionales (Jiménez, 2007) y son de bajo costo (Bojórquez et al., 2015). No obstante, se requiere cosechar y transportar el pasto hasta los galpones o ambientes donde se alojan estos animales, actividad que requiere de mano de obra, costo que representa el 20\% del costo de producción (R. Jiménez, Jauja, comunicación personal). 
El costo de la mano de obra empleada en actividades agropecuarias ha aumentado en el tiempo, en correspondencia con los incrementos de la remuneración mínima vital (BCRP, 2020) con tendencia a seguir subiendo. Este incremento en los costos, sumado al comportamiento estable del precio del cuy en los últimos cinco años, por un posible equilibrio entre oferta y demanda, está afectando la rentabilidad de los sistemas de producción de cuyes y a futuro es un factor que puede poner en riesgo la sostenibilidad económica de estos sistemas de producción.

El desarrollo de un sistema de pastoreo en cuyes se presenta como una alternativa tecnológica al sistema de crianza en galpones, con ventajas en la disminución de los costos de producción, debido a que no se requiere de un galpón, ni equipos para la cosecha y trasporte de pastos, ni mano de obra para cosecha y trasporte de pastos, limpieza de galpones y manejo de estiércol (Aliaga et al., 2009). Adicionalmente, los sistemas pastoriles pueden proporcionar un medio ecológicamente sostenible, así como contribuir al bienestar animal (Lund, 2006; Alonso, 2016). En ese sentido, existe una alta demanda de productos naturales, mayormente en el mercado internacional, donde los consumidores no solo aprecian la calidad del producto, sino el proceso productivo que toma en cuenta el bienestar animal. Un ejemplo de esta tendencia es que existen consumidores dispuestos a pagar un mayor precio por carne de cerdos criados al pastoreo (Pietrosemoli, 2016).

Hace 40 años se desarrolló una propuesta tecnológica de pastoreo de cuyes, empleando jaulas móviles de $1.2 \mathrm{~m}^{2} \mathrm{y}$ suplementación con concentrado, que, en contraste con la crianza en galpones, mostró ventajas económicas y de manejo (Aliaga et $a l ., 2009)$, pero su aceptación fue muy limitada por parte de los productores, probablemente porque en ese momento las ventajas de la tecnología no resultaron convincentes. En los últimos años se ha iniciado el desarrollo de un sistema pastoril en cuyes, como una propuesta tecnológica diferente, que incluye la producción, sostenibilidad, eficiencia, rentabilidad, bienestar animal, equilibrio ecológico y participación de los productores. En ese proceso, Mamani et al. (2015) determinaron el periodo de descanso de los pastos en 46 días. En este sentido, el presente estudio hace una primera aproximación de carga animal óptima de cuyes al pastoreo en función al rendimiento de pastura, parámetros productivos y económicos, al comparar cuatro niveles de carga animal durante la época lluviosa en la Sierra del Perú.

\section{Materiales Y MéTodos}

\section{Lugar del Estudio}

El estudio se desarrolló entre noviembre de 2017 y abril de 2018 en la Unidad de Investigación en Cuyes de la Estación IVITA El Mantaro, Facultad de Medicina Veterinaria, Universidad Nacional Mayor de San Marcos, localizada en la margen izquierda del río Mantaro, en el distrito de El Mantaro, provincia de Jauja, región Junín a $3320 \mathrm{~m}$ de altitud. La zona presenta una precipitación media anual de $704 \mathrm{~mm}$ y una temperatura media anual de $11.9^{\circ} \mathrm{C}$ (mínimo 4.1 y máximo $\left.19.1{ }^{\circ} \mathrm{C}\right)$ (IGP, 2005).

\section{Animales y Pastura}

Se emplearon 132 cuyes G (Jiménez y Huamán, 2010), machos destetados de aproximadamente 15 días de edad y pesos iniciales similares. Los cuyes fueron distribuidos en tres grupos de 44 animales para cada ciclo de engorde de 60 días, desarrollados secuencialmente. Los 44 cuyes de cada ciclo se repartieron aleatoriamente en grupos de $8,10,12$ y 14 cuyes para los tratamientos T1, T2, T3 y T4, respectivamente, de tal manera que se represente la carga animal correspondiente para cada tratamiento. El ciclo de engorde de 60 días corresponde al periodo que los cuyes requieren pastorear para llegar al peso comercial cercano a $1 \mathrm{~kg}$. 


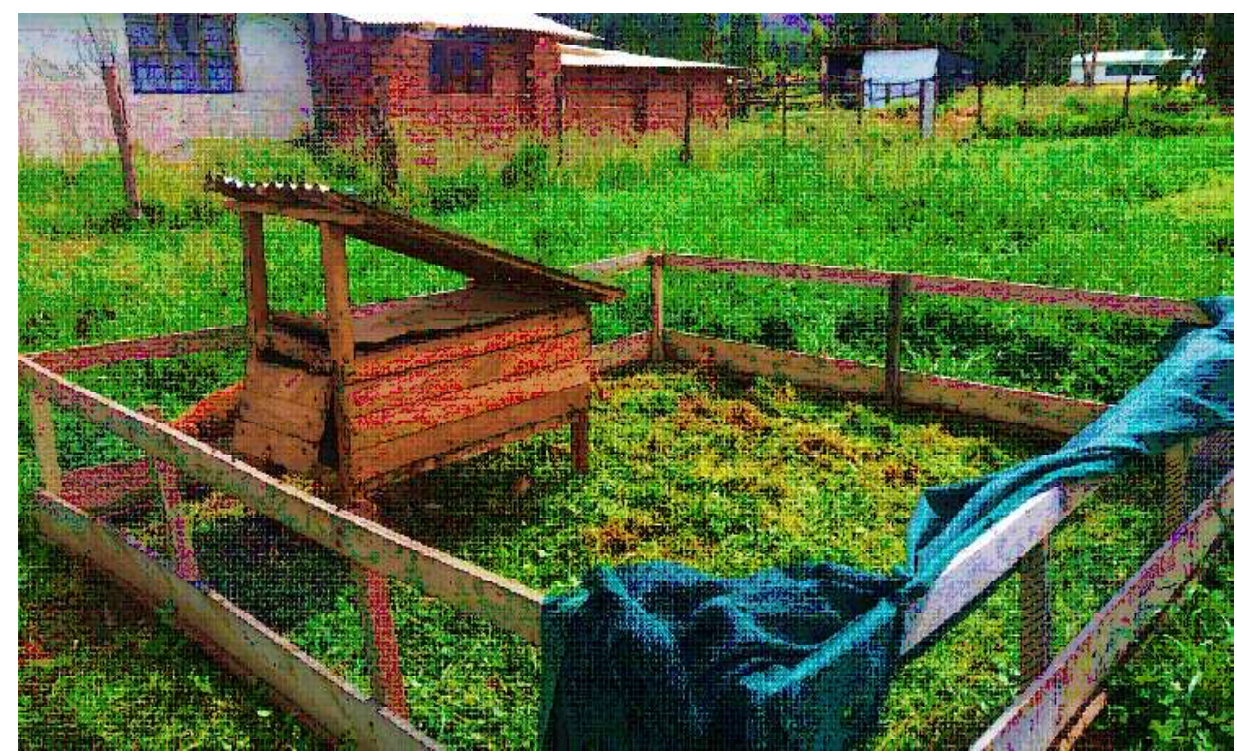

Figura 1.Cerco móvil para un sistema de pastoreo en cuyes. Obsérvese la madriguera dentro del área cercada

En un área de aproximadamente $1000 \mathrm{~m}^{2}$ se instalaron pastos asociados, compuestos por gramíneas y leguminosas. Se empleó la siguiente densidad de siembra para lograr una variada composición botánica: Lolium multiflorum (900 g), Lolium perenne (900 g), Dactylis glomerata (300 g), Trifolium pratense $(200 \mathrm{~g})$, Trifolium repens $(200 \mathrm{~g}) \mathrm{y}$ Medicago sativa variedad CUF 101 (500 g).

\section{Instalaciones}

Se delimitaron áreas contiguas de 200 $\mathrm{m}^{2}$ para cada tratamiento, las cuales fueron asignadas de manera aleatoria. Cada área contó con un cerco cuadrangular móvil de pastoreo de $8.33 \mathrm{~m}^{2}(3 \times 2.78 \mathrm{~m})$ y $0.6 \mathrm{~m}$ de altura, construido de madera de eucalipto y recubierto por los costados con una malla metálica cuadrada de 1 pulgada. La parte superior fue cubierta con malla raschel (Figura 1).

En el centro de cada cerco se ubica una madriguera elaborada con madera de eucalipto $(0.5 \mathrm{~m}$ de ancho, $1 \mathrm{~m}$ de largo y
$0.3 \mathrm{~m}$ de alto), recubierta en la parte superior con calamina. La madriguera contaba con una entrada de $15 \mathrm{~cm}$ de diámetro junto a una rampa y un orificio para la ventilación: asimismo, listones de madera a manera de patas que elevaban la madriguera a $20 \mathrm{~cm}$ del piso (Figura 1). En el piso interno de las madrigueras se aplicaba cal y un poco de forraje seco a manera de cama, cada dos semanas. El uso de la madriguera tiene por finalidad proporcionar seguridad ante el acecho de depredadores y cobijo durante las noches y en momentos de condición climática adversa.

\section{Diseño Experimental}

Se utilizó un diseño de bloques completos al azar con tres ciclos de engorde como bloque y cuatro tratamientos correspondientes a cuatro niveles de carga animal: 400 (T1), 500 (T2), 600 (T3) y 700 (T4) cuyes/ha/año, empleando áreas de $200 \mathrm{~m}^{2}$, donde pastorearon grupos de $8,10,12$ y 14 cuyes respectivamente. Los bloques corresponden a tres 
ciclos de engorde de 60 días cada uno. Las unidades experimentales difieren en el número de animales por grupo, necesario para establecer los niveles de carga animal para cada tratamiento, de tal manera que se usa la misma área para cada tratamiento (Lascano et al., 1986). Las cargas animales empleadas fueron equidistantes para un adecuado análisis por contrastes ortogonales lineal y cuadrático, partiendo de la referencia de 555 cuyes/ha/año utilizada por Mamani et al. (2015).

El ensayo se inició a finales de julio de 2018 con la instalación de la pastura. El primer ciclo de engorde se inició a los tres meses de sembrada la pastura. Debido a que en los primeros 46 días del primer ciclo de engorde no se cumplía con los 46 días de periodo de descanso, no se consideró el dato de rendimiento de la pastura en este primer ciclo. La pastura fue fertilizada con $10 \mathrm{~kg}$ de fosfato diamónico y $5 \mathrm{~kg}$ de cloruro de potasio a la siembra, repitiéndose en noviembre y marzo.

Los tratamientos fueron distribuidos aleatoriamente en las cuatro áreas de pastoreo de $200 \mathrm{~m}^{2}$, en cada ciclo de engorde. Dentro de cada área de $200 \mathrm{~m}^{2}$ se manejaron potreros de $8.33 \mathrm{~m}^{2}$, donde cada grupo de cuyes pastorearon durante 48 horas iniciando a las 14:00 horas, luego se rotaba al potrero contiguo y así sucesivamente hasta completar un ciclo de engorde de 60 días. En cada ciclo de engorde se realizaban 30 rotaciones de potrero; es decir, se utilizaban los $200 \mathrm{~m}^{2}$ de pastura en 24 rotaciones más 6 rotaciones adicionales.

Al inicio de cada ciclo de engorde (14:00 h), se registró el peso inicial individual de los cuyes, empleando una balanza electrónica de $1 \mathrm{~g}$ de sensibilidad. El peso final se tomó al concluir el ciclo de sesenta días de engorde. Para la estimación de producción de materia seca (MS) de la pastura se tomaron muestras de pasto ofrecido y rechazado durante el ciclo de engorde, utilizando un cuadrante de metal de $0.0625 \mathrm{~m}^{2}$ área (Cangiano, 1996), cortando la pastura a ras del cuadrante con una hoz. Transcurrido 48 horas se vuelve a obtener una muestra representativa del pasto rechazado en el potrero, empleando el mismo cuadrante y realizando el mismo tipo de corte. Este procedimiento se realizó en cada rotación del potrero de cada tratamiento.

Toda muestra de pastura ofrecida y rechazada fue pesada y registrada después del corte, empleando la balanza indicada previamente. De cada muestra se tomó una submuestra representativa para la estimación de la MS. El material restante fue retornado al potrero para no afectar el consumo. Las submuestras fueron llevadas al Laboratorio de Nutrición de la Estación IVITA El Mantaro, y secadas en una estufa a $60^{\circ} \mathrm{C}$ por $48 \mathrm{~h}$ (De La Roza-Delgado et al., 2002) para la determinación de la MS.

\section{Variables de Respuesta Evaluadas}

Rendimiento de pastura. El rendimiento de pastura mide la cantidad de MS que produce la pastura luego de 46 días de descanso. Se expresa en g MS $/ \mathrm{m}^{2} /$ día. Se determinó multiplicando el peso de la muestra de pasto ofrecido, expresado en gramos, por la proporción de materia seca, multiplicado por 16 para obtener el rendimiento en metro cuadrado y dividido entre 46, el periodo de descanso de la pastura, para obtener el rendimiento por día.

Pastura consumida respecto a la oferta. Esta variable cuantifica la proporción de pastura consumida con respecto a la ofrecida. Se cuantificó por diferencia entre pastura ofrecida y la rechazada, dividido entre la cantidad de pastura ofrecida.

Peso inicial y final por cuy. Es el peso promedio individual de los cuyes de cada tratamiento, registrado al inicio y final del ciclo de engorde. 
Cuadro 1. Efecto de la carga animal de cuyes sobre la producción y uso de una pastura mixta

\begin{tabular}{lccccc}
\hline $\begin{array}{l}\text { Carga animal } \\
\text { (cuyes/ha/año) }\end{array}$ & 400 & 500 & 600 & 700 & p-valor \\
\hline $\begin{array}{l}\text { Rendimiento de pastura }\left(\mathrm{g} \mathrm{MS} / \mathrm{m}^{2} / \mathrm{dí}\right) \\
\begin{array}{l}\text { Proporción de pastura consumida } \\
\text { respecto a la oferta }\end{array}\end{array}$ & 8.92 & 8.62 & 11.03 & 10.43 & 0.298 \\
\hline
\end{tabular}

Ganancia de peso vivo individual. Corresponde a la diferencia entre peso final individual y peso inicial individual, dividido entre el número de días del ciclo de engorde (60). Se expresa en g/cuy/día.

Consumo de alimento individual. Este valor se obtuvo por diferencia entre el pasto ofrecido y rechazado, en base seca, de cada periodo de pastoreo de 48 horas y promediando los datos de las 30 rotaciones de un ciclo de engorde, dividido entre dos para obtener la cantidad de alimento consumido por día y después dividido entre el número de cuyes que pastoreaban en cada tratamiento, para obtener el consumo promedio individual por unidad de tiempo (g MS/cuy/día).

Índice de conversión alimenticia (ICA). Este valor es un cociente que resulta al dividir el consumo de alimento individual entre la ganancia de peso vivo individual. Este parámetro indica lo que se consume para incrementar un kilo de peso vivo.

Ganancia de peso por unidad de área. Se determinó por la multiplicación de ganancia de peso individual diario por el número de animales que pastorean en el potrero, dividido entre el área pastoreada total $\left(200 \mathrm{~m}^{2}\right) \mathrm{y}$ se expresa en $\mathrm{g} / \mathrm{m}^{2} /$ día.

Costo de producción unitario. El costo de producción fue estimado por la suma de costos fijos (terreno, preparación de terreno, semillas de pastos, fertilizantes, cerco perimé- trico, cerco de potreros, madrigueras y mano de obra para traslado de cercos) y variables (precio de cuyes destetados) proyectados para una hectárea. El cálculo se realizó para cada tratamiento de carga animal por el periodo de un semestre, tiempo de evaluación del presente estudio, dividido entre la cantidad de cuyes producidos en el semestre.

Ratio beneficio costo. El ratio beneficio costo se obtuvo al dividir el precio de venta del cuy, equivalente a S/. 18.00, entre su costo de producción unitario.

\section{Análisis de Datos}

Los datos de las variables de respuesta fueron evaluados mediante análisis de varianza aplicado a un diseño de bloques completos al azar, empleando contrastes ortogonales lineal y cuadrático. Se utilizó el paquete estadístico SAS (SAS Institute, 1990).

\section{Resultados y Discusión}

Efecto de la carga animal sobre el rendimiento de la pastura

El rendimiento de la pastura representa la oferta de alimento para los cuyes y no se vio afectado por la carga animal (Cuadro 1). Ninguna de las cargas utilizadas perjudicó el rendimiento de la pastura como ocurre cuan- 
Cuadro 2. Consumo promedio individual en tres periodos del ciclo de engorde ( $\mathrm{g}$ MS/cuy/día) en cuyes al pastoreo

\begin{tabular}{ccccc}
\hline \multirow{2}{*}{ Periodo } & \multicolumn{5}{c}{$\begin{array}{c}\text { Carga animal } \\
\text { (cuyes/ha/año) }\end{array}$} \\
\cline { 2 - 5 } & 400 & 500 & 600 & 700 \\
\hline Días 1-20 & 93.4 & 81.6 & 87.3 & 71.1 \\
Días 21-40 & 90.3 & 88.0 & 78.8 & 97.5 \\
Días 41-60 & 105.3 & 90.1 & 88.9 & 91.1 \\
\hline
\end{tabular}

No hubo diferencias estadísticas significativas $(p>0.05)$ entre cargas animales

Cuadro 3. Efecto de la carga animal sobre parámetros productivos de cuyes en pastoreo

\begin{tabular}{lccccc}
\hline & \multicolumn{3}{c}{ Carga animal (cuyes/ha/año) } & \multirow{2}{*}{ p-valor } \\
\cline { 2 - 5 } & 400 & 500 & 600 & 700 & \\
\hline Peso inicial $(\mathrm{g})$ & 372.3 & 374.0 & 373.3 & 373.3 & 0.250 \\
Peso final (g) & 1019.8 & 907.3 & 994.2 & 906.3 & 0.137 \\
$\begin{array}{l}\text { Ganancia de peso } \\
\text { (g/cuy/día) }\end{array}$ & 10.80 & 8.87 & 10.33 & 8.873 & 0.124 \\
$\begin{array}{l}\text { Consumo de alimento } \\
\text { (g/cuy/día) }\end{array}$ & 96.37 & 86.57 & 84.00 & 86.57 & 0.610 \\
ICA & 9.0 & 9.8 & 8.03 & 9.67 & 0.299 \\
\hline
\end{tabular}

do hay sobrepastoreo (Romero et al., 2010). Además, la pastura utilizada tuvo las condiciones de alta productividad y mejor tolerancia al pastoreo de los cuyes debido a que la evaluación se hizo con una pastura asociada joven y en la estación lluviosa (Bojórquez et al., 2015).

El rendimiento promedio reportado en la pastura fue ligeramente superior al obtenido por Mamani et al. (2015) y asumiendo que las pasturas producen el doble durante la época lluviosa de lo producido en época seca (Bojórquez, 1998), la producción estimada tendría niveles de 23159 a $29634 \mathrm{Kg}$ de MS/ha/año para las parcelas utilizadas en los cuatro tratamientos. Esta proyección presagia una alta producción, que podría superar a lo reportado por Bojórquez (1998).

Una de las posibles razones para no hallar efecto de la carga animal sobre el rendimiento de la pastura es que la presión de pastoreo no fue lo suficientemente alta para generar deterioro de la pastura (Holechek et al., 2011), efecto que ocurre cuando después del pastoreo la altura de los pastos es menor a $5 \mathrm{~cm}$ (Romero et al., 2010). 
Se presumía que al incrementar el peso vivo inicial en 2.56 veces para llegar al peso vivo final (Cuadro 3) pudiera influir en el incremento del consumo de alimento conforme los animales aumentaban de peso y debería haber un mayor consumo hacia el final del ciclo de engorde (Ramírez, 2009). Sin embargo, los cuyes mostraron un consumo variable no ajustado a una proporción fija respecto al peso vivo (Cuadro 2), lo cual indica que la presión de pastoreo estuvo poco influenciada por las variaciones de peso vivo de los cuyes.

Efecto de la carga animal sobre la respuesta productiva

La respuesta productiva de los cuyes no se afectó por efecto de la carga animal (Cuadro 3). Al respecto, se puede deducir que las ofertas de alimento de las distintas cargas animales permitieron consumos de alimento, ganancias de peso y conversiones alimenticias similares, de allí que la capacidad de carga de la pastura utilizada en el presente estudio sea probablemente mayor a 700 cuyes/ha/año.

El efecto de la carga animal supone un incremento en la ganancia de peso individual con cargas bajas, hasta un límite definido por la disminución en la calidad de los pastos por maduración. A partir de esta máxima ganancia de peso individual el incremento en la carga animal va reduciendo la ganancia de peso individual de manera lineal. En paralelo, la ganancia por unidad de área se incrementa hasta cierto límite para luego disminuir junto a la ganancia de peso individual. De acuerdo con este patrón de comportamiento de los datos la carga animal óptima estaría definida por la intersección entre la regresión lineal de ganancia de peso individual y la regresión cuadrática de la ganancia de peso por unidad de área (Aiken, 2016; Melvin et al., 2020).
Las ganancias de peso por cuy no mostraron diferencias estadísticas ni tampoco una tendencia decreciente, lo cual indica que las cargas animales utilizadas fueron insuficientes para mostrar efecto sobre la ganancia de peso. Es probable que al utilizar cargas más elevadas pueda describirse un comportamiento lineal descendente en la ganancia de peso individual de los cuyes.

Hay evidencia que la proporción de leguminosa dentro de la composición botánica de la pastura tiene efecto sobre la relación ganancia de peso y carga animal, debido a que la disponibilidad mínima de ciertas leguminosas limita la producción animal (Hernández et al., 1995; Pereira et al., 2019). Al respecto, en el presente estudio, la siembra de la pastura se hizo al voleo y es posible que la proporción gramínea leguminosa no haya sido homogénea entre los tratamientos, dado que no se hizo la medición de la composición botánica.

Si bien la carga animal afectó significativamente y de forma lineal la respuesta productiva por unidad de área $(\mathrm{p}<0.05)$ (Cuadro 4), no llega a mostrarse el comportamiento real de la variable que debió ser una regresión cuadrática (Aiken, 2016; Melvin et al., 2020), lo cual podría evidenciarse cuando se evalúe cargas más altas.

Cuadro 4. Efecto de la carga animal sobre la ganancia de peso por unidad de área

\begin{tabular}{cc}
\hline $\begin{array}{c}\text { Carga animal } \\
\text { (cuyes/ha/año })\end{array}$ & $\begin{array}{c}\text { Ganancia de peso } \\
\left(\mathrm{g} / \mathrm{m}^{2} / \mathrm{dí}^{1}\right)^{1}\end{array}$ \\
\hline 400 & 0.43 \\
500 & 0.44 \\
600 & 0.62 \\
700 & 0.62 \\
\hline$p$-valor & 0.01 \\
\hline
\end{tabular}

${ }^{1}$ Efecto lineal $(p<0.05)$ 
Cuadro 5. Efecto de la carga animal sobre parámetros económicos de cuyes en pastoreo

\begin{tabular}{lccccc}
\hline & \multicolumn{3}{c}{ Carga animal (cuyes/ha/año) } & \multirow{2}{*}{ p-valor } \\
\cline { 2 - 5 } & 400 & 500 & 600 & 700 & \\
\hline $\begin{array}{l}\text { Costo de producción unitario } \\
\left(\mathrm{S} / \text {. }^{1}\right.\end{array}$ & 17.98 & 15.78 & 14.32 & 13.27 & 0.0001 \\
Ratio beneficio costo $^{1}$ & 0.97 & 0.96 & 1.27 & 1.23 & 0.0046 \\
\hline
\end{tabular}

${ }^{1}$ Efecto lineal $(p<0.05)$

Muchas de las variables biológicas, como la ganancia de peso por unidad de área, cuando se distribuyen en función a la carga animal suelen ajustarse mejor a una regresión de tipo cuadrática (Melvin et al., 2020) y en evaluaciones de carga animal, la ganancia de peso por unidad de área suele tener ese comportamiento. No obstante, las cargas utilizadas en el presente estudio fueron limitadas para describir un efecto cuadrático.

Efecto de la carga animal sobre parámetros económicos

Se encontró un efecto de la carga animal sobre los parámetros económicos de cuyes en pastoreo $(p<0.05$; Cuadro 5), donde el menor costo de producción por cuy corresponde a la carga de 700 cuyes/ha/año. El ratio beneficio costo también mostró el efecto de la carga animal $(\mathrm{p}<0.05)$ y un efecto lineal en los tratamientos (Cuadro 5). Cabe mencionar que los resultados están influenciados por la diferencia en el número de animales entre los tratamientos, para generar las cargas respectivas, y no tienen carácter determinante porque las variables productivas, especialmente la ganancia de peso, no mostraron efecto de la carga animal.

El análisis económico permite pronosticar si la tecnología puede aportar retorno económico al sistema productivo que se desarrolle, en este caso, pastoreo en cuyes, Sin embargo, dado que los datos no se ajustan a una regresión cuadrática no es posible determinar la carga animal óptima que maximice el ratio beneficio costo.

\section{Conclusiones}

- Las cargas animales entre 400 a 700 cuyes/ha/año no mostraron efecto sobre las variables rendimiento de pastura, ganancia de peso individual, consumo de alimento e índice de conversión alimenticia en cuyes al pastoreo durante la época lluviosa.

- El rango de carga animal empleada en el estudio fue insuficiente para determinar una carga animal óptima.

- Por lo pronto, se puede utilizar la carga animal de 700 cuyes/ha/año porque permite lograr una mayor cantidad de cuyes con parámetros productivos similares a cargas menores.

\section{Literatura Citada}

1. Aiken GE. 2016. Grazing management options in meeting objectives of grazing experiments. Applied Anim Sci 32: 1-9. doi: 10.15232/pas.2015-01406

2. Aliaga L, Moncayo $R$, Rico $E$, Caycedo A. 2009. Producción de cuyes. Lima: Fondo Editorial de la Universidad Católica Sedes Sapientiae. 808 p. 
3. Alonso J. 2016. Principios agroecológicos en tecnologías cubanas con leguminosas para la producción animal. Cuban J Agr Sci 50: 171-183.

4. [BCRP] Banco Central de Reserva del Perú. 2020. Remuneración mínima vital nominal. BCRPData. Lima. Perú. [Internet]. Disponible en: https:/ /estadisticas.bcrp.gob.pe/estadisticas/ series/mensuales/resultados/ $\mathrm{PN} 02124 \mathrm{PM} / \mathrm{html}$

5. Bojórquez C.1998. Producción de pastos cultivados en tres zonas agroecológicas de la sierra central. Rep Inv Pec IVITA 9: 20-31.

6. Bojórquez C, Rojas JD, Ordoñez JH. 2015. Pastos cultivados en el valle del Mantaro. Lima: UNMSM. 147 p.

7. Cangiano C.1996. Métodos de medición de fitomasa aérea. En: Producción animal en pastoreo Buenos Aires, Argentina: INTA. p 117-128.

8. Chauca L. 1997. Producción de cuyes (Cavia porcellus). Roma: FAO. 77 p.

9. De La Roza-Delgado B, Martínez A, Argamentería A. 2002. Determinación de materia seca en pastos y forrajes a partir de la temperatura de secado para análisis. Pastos 32: 91-104.

10. Hernández M, Argel PJ, Ibrahim MA, 'T Mannetje L. 1995. Pasture production, diet selection and live weight gains of cattle grazing Brachiaria brizantha with or without Arachis pintoi at two stocking rates in the Atlantic Zone of Costa Rica. Trop Grasslands 29: 134-141.

11. Holechek J, Pieper R, Herbel C. 2011. Range management: principles and practices. $6^{\text {th }}$ ed. New Jersey, USA: Prentice Hall. 444 p.

12. [IGP] Instituto Geofísico del Perú. 2005. Atlas climático de precipitación y temperatura del aire de la cuenca del Río Mantaro. Lima: CONAM. 107 p.

13. [INEI] Instituto Nacional de Estadística e Informática. 2012. IV Censo Nacional Agropecuario. Lima. [Internet]. Disponible en : http://censos.inei.gob.pe/ Cenagro/redatam/
14. [INEI] Instituto Nacional de Estadística e Informática. 2017. Encuesta Nacional Agropecuaria. Lima. [Internet]. Disponible en: https://www.inei.gob.pe/ media/MenuRecursivo/publicaciones_digitales/Est/Lib1593

15. Jiménez R. 2007. Uso de insumos agrícolas locales en la alimentación de cuyes en valles interandinos. Arch Latinoam Prod Anim 15 (Supl 1): 229-232.

16. Jiménez R, Huamán A. 2010. Manual para el manejo de reproductores híbridos especializados en producción de carne. El Mantaro, Perú: INCAGRO ACRICUCEN- UNMSM. 109 p.

17. Lascano C, Pizarro E, Toledo JM. 1986. Recomendaciones generales para evaluar pasturas con animales. En: Lascano C, Pizarro E (eds). Evaluación de pasturas con animales. Alternativas metodológicas. Colombia: RIEPT CIAT. $292 \mathrm{p}$.

18. Lund V. 2006. Natural living - a precondition form animal welfare in organic farming. Livest Sci 100: 71-83. doi: 10.1016/j.livprodsci.2005.08.005

19. Mamani R, Jiménez R, San Martín F, Ara M. 2015. Determinación del periodo óptimo de descanso de la pastura asociada Loliumultiflorum, Trifulium pratense y Medicago sativa, pastoreada por cuyes en la sierra central del Perú. Rev Inv Vet Perú 26: 404-411. doi: 10.15381/rivep.v26i3.11174

20. Melvin GR, Frost W, McDougald N. 2020. Grazing management. In: Melvin G (ed). Ecology and management of annual rangelands series. Davis, California: University of California. p 1-25.

21. Pereira JM, Rezende CP, Ferreira AM, Homem BGC, Casagrande DR, Macedo TM, Alves BJR, et al. 2019. Production of beef cattle grazing on Braquiaria brizantha (Marandu grass) - Arachis pintoi (forage peanut cv. Belomonte) mixtures exceeded that on grass monocultures fertilized with $120 \mathrm{~kg}$ N/ha. Grass Forage Sci 75: 28-36. doi: 10.1111/gfs. 12463 
22. Pietrosemoli S. 2016. Porcinos al pastoreo, estrategias para reducir su potencial impacto ambiental. Arch Latinoam Prod Anim 24: 89-94.

23. Ramírez RG 2009. Nutrición de rumiantes. Sistemas extensivos. México. Ed Trillas. $314 \mathrm{p}$.
24. Romero O, Rodríguez J, Gutierrez E, Colín J. 2010. Sistemas de pastoreo. En: Rodríguez J, Gutierrez E, Rodríguez H (eds). Dinámica de sistemas de pastoreo. México: Ed Trillas. p 45-59.

25. SAS Institute. 1990. User's Guide, v. 6. $4^{\text {th }}$ ed. Cary, NC: SAS Institute Inc. 943 p. 